\title{
Astragaloside IV prevents damage to human mesangial cells through the inhibition of the NADPH oxidase/ROS/Akt/NF-kB pathway under high glucose conditions
}

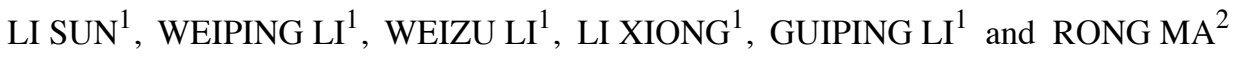 \\ ${ }^{1}$ Department of Pharmacology, College of Basic Medicine, Anhui Medical University, Hefei, \\ Anhui 230032, P.R. China; ${ }^{2}$ Department of Integrative Physiology and Cardiovascular Research Institute, \\ University of North Texas Health Science Center, Fort Worth, TX 76107, USA
}

Received January 20, 2014; Accepted April 1, 2014

DOI: $10.3892 /$ ijmm.2014.1741

\begin{abstract}
Glomerular hypertrophy and hyperfiltration are the two major pathological characteristics of the early stages of diabetic nephropathy (DN), which are respectively related to mesangial cell (MC) proliferation and a decrease in calcium influx conducted by canonical transient receptor potential cation channel 6 (TRPC6). The marked increase in the production of reactive oxygen species (ROS) induced by hyperglycemia is the main sponsor of multiple pathological pathways in DN. Nicotinamide adenine dinucleotide phosphate (NADPH) oxidase is an important source of ROS production in MCs. Astragaloside IV (AS-IV) is an active ingredient of Radix Astragali which has a potent antioxidative effect. In this study, we aimed to investigate whether high glucose (HG)-induced NADPH oxidase activation and ROS production contribute to MC proliferation and the downregulation of TRPC6 expression; we also wished to determine the effects of AS-IV on MCs under HG conditions. Using a human glomerular mesangial cell line, we found that treatment with AS-IV for $48 \mathrm{~h}$ markedly attenuated HG-induced proliferation and the hypertrophy of MCs in a dose-dependent manner. The intracellular ROS level was also markedly reduced following treatment with AS-IV. In addition, the enhanced activity of NADPH oxidase and the expression level of NADPH oxidase 4 (Nox4) protein were decreased. Treatment with AS-IV also inhibited the phosphorylation level of Akt and IкB $\alpha$ in the MCs. In addition, TRPC6 protein expression and the intracellular free calcium concentration were also markedly reduced following treatment with AS-IV under HG conditions. These results suggest
\end{abstract}

Correspondence to: Professor Weiping Li, Department of Pharmacology, College of Basic Medicine, Anhui Medical University, 81 Meishan Road, Hefei, Anhui 230032, P.R. China

E-mail:1wp19@126.com

Key words: diabetic nephropathy, human mesangial cells, nicotinamide adenine dinucleotide phosphate oxidase, reactive oxygen species, astragaloside IV that AS-IV inhibits HG-induced mesangial cell proliferation and glomerular contractile dysfunction through the NADPH oxidase/ROS/Akt/nuclear factor- $\kappa \mathrm{B}(\mathrm{NF}-\kappa \mathrm{B})$ pathway, providing a new perspective for the clinical treatment of $\mathrm{DN}$.

\section{Introduction}

Diabetic nephropathy (DN) is one of the main chronic complications of diabetes mellitus (DM) associated with capillaries, often leading to chronic renal failure and end-stage renal disease (1). Although the precise pathogenesis of DN has not been elucidated, a number of studies have demonstrated that glomerular hyperfiltration and mesangial expansion are the two characteristic pathophysiological changes occurring during the early stages of DN $(2,3)$.

Mesangial cells (MCs), located within glomerular capillary loops, play an important role in the regulation of glomerular hemodynamics due to their contractile function (4). There is increasing evidence indicating that hyperglycemia directly stimulates MCs, which subsequently results in mesangial contractile dysfunction and glomerular hyperfiltration $(5,6)$. The hypocontractility of MCs is closely related to the reduced calcium influx $(7,8)$.

Canonical transient receptor potential cation channels (TRPCs), as members of the transient receptor potential (TRP) superfamily, are $\mathrm{Ca}^{2+}$ permeable cation channels widely expressed in a series of tissues and cells $(9,10)$. The TRPC family includes seven related members, designated as TRPC1-7 (11). Among these, TRPC6 is closely associated with kidney disease $(12,13)$. Möller et al (14) found that TRPC6 exists throughout the glomerulus in kidney tissues, particularly in MCs. Additionally, it has been dcemonstrated that hyperglycemia downregulates the expression of TRPC6 protein, which results in a decrease in intracellular calcium, leading to impaired MC contractile response and glomerular hyperfiltration (15). However, the underlying molecular mechanisms of TRPC6 protein downregulation in MCs have not yet been fully elucidated.

Hyperglycemia can also cause the abnormal proliferation of MCs in DN, leading to glomerular hypertrophy and fibrosis (16). Clinical and animal experiments have demonstrated an increase 
in the production of reactive oxygen species (ROS) in MCs, as the direct result of chronic exposure to high glucose $(\mathrm{HG})(17,18)$. The overproduction of ROS can transduce and amplify glucose signaling, playing a key role in MC proliferation (19). ROS can also modulate $\mathrm{Ca}^{2+}$ channels by activating various signaling cascades (20).

In the kidneys, particularly in MCs, nicotinamide adenine dinucleotide phosphate (NADPH) oxidase is the predominant source of ROS (21). The phagocyte NADPH oxidase consists of two membrane-associated subunits, $\mathrm{p} 22^{\text {phox }}$ and gp $91^{\text {phox }}$, and at least four cytoplasmic components, $\mathrm{p} 47^{\text {phox }}, \mathrm{p} 67^{\text {phox }}, \mathrm{p} 40^{\text {phox }}$ and the small GTPase, Rac. NADPH oxidase in the resting state becomes activated upon interaction between the catalytic core (membrane-associated subunits) and the cytosolic regulatory subunits (22). There are six homologues of phagocytic gp91 ${ }^{\text {phox }}$ proteins expressed by distinct non-phagocytic cells (23). It has been reported that NADPH oxidase 4 (Nox4) is the key subunit of NADPH oxidase expressed in MCs, and Nox4-derived ROS is the major contributor to renal morphological changes and functional abnormalities in DN (24).

Akt, also known as protein kinase B (PKB), belonging to serine/threonine kinase family members, is one of the downstream effectors of phosphoinositide 3-kinase (PI3K) which participates in numerous signaling pathways involved in diverse physiological processes, including glucose metabolism, protein synthesis, cell proliferation, cell apoptosis $(25,26)$. Kim et al reported that Akt was activated in renal damage in streptozotocin-induced diabetic mice (27). It has also been demonstrated that Akt is an important mediator of MC proliferation, and can be regulated by ROS (28).

Akt is not only the downstream signaling molecule of PI3K, but also the major upstream element in the activation of nuclear factor- $\kappa \mathrm{B}(\mathrm{NF}-\kappa \mathrm{B})$. Akt promotes the transcriptional activity of NF- $\kappa \mathrm{B}$ through a variety of mechanisms and the signaling cascade eventually leads to cell proliferation and migration (29). A number of studies have demonstrated that NF- $\kappa \mathrm{B}$ is activated in MCs by hyperglycemia, and plays a crucial role in the progression of DN $(30,31)$. In addition, a recent study found that ROS is the important messenger in the NF- $\mathrm{NB}$ signaling pathway (32). Of note, the promoter region of TRPC6 has NF- $\kappa$ B binding sites (33). Therefore, we hypothesized that the redox-sensitive $\mathrm{NF}-\kappa \mathrm{B}$ participates in the downregulation of TRPC6 in DN. Thus, we aimed to explore whether NADPH oxidase-derived ROS is involved in HG-induced cell proliferation and the downregulation of TRPC6 in MCs through the Akt/NF- $\mathrm{kB}$ pathway.

Astragaloside IV (AS-IV, 3-O- $\beta$-D-xylopyranosyl-6-O- $\beta$ D-glucopyranosylcycloastragenol), a purified small molecular saponin, is one of the main active ingredients of Radix Astragali, which has been reported to possess comprehensive biological properties, including antioxidant, anti-inflammatory, immunoregulatory and anti-aging properties and to improve intellectual development (34). A recent studies indicated that AS-IV ameliorates proteinuria in rats with adriamycin nephropathy (35). It has also been reported that AS-IV significantly inhibits renal oxidative stress and apoptosis in STZ-induced diabetic rats (36). However, the protective effects and the precise mechanisms of action of AS-IV on oxidative stress-induced injury in MCs under HG conditions have not yet been fully elucidated.

The present study aimed to investigate the effects of AS-IV on HG-induced MC proliferation and the downregulation of
TRPC6 through a mechanism associated with the inhibition of NADPH oxidase-mediated ROS production, Akt and $\mathrm{NF}-\kappa \mathrm{B}$ activation, in an attempt to provide a novel therapeutic approach for the treatment of DN.

\section{Materials and methods}

Reagents. Dulbecco's modified Eagle's medium (DMEM) and fetal bovine serum (FBS) were purchased from HyClone Laboratories, Inc. (Logan, UT, USA). Penicillin/streptomycin solution, $0.05 \%$ Trypsin-EDTA, phoshate-buffered saline (PBS), and dimethyl sulfoxide (DMSO) were purchased from Invitrogen Life Technologies (Carlsbad, CA, USA). 3-(4,5-Dimethylthiazol2-yl)-2,5-diphenyl tetrazolium bromide (MTT), $\alpha$-mannitol (MA), diphenyleneiodonium (DPI), tempol, LY294002, sulfasalazine (Sul), Fluo3/AM ester, and 2',7'-dichlorofluorescein diacetate (DCFH-DA) were obtained from Sigma (St. Louis, MO, USA). The cell NADPH oxidase colorimetric assay kit was purchased from GenMed Scientifics Inc. (Boston, MA, USA). Antibodies were obtained from the following sources: anti-Nox4 polyclonal antibody (pAb), anti-phospho-Akt pAb and anti-Akt pAb were from Santa Cruz Biotechnology, Inc. (Santa Cruz, CA, USA); anti-phospho-IкB $\alpha$ monoclonal antibody $(\mathrm{mAb})$ and anti-I $\mathrm{B} \alpha \mathrm{mAb}$ were from Cell Signaling Technology, Inc. (Beverly, MA, USA); anti-TRPC6 mAb was from Abcam (Cambridge, UK); anti- $\beta$-actin mAb, horseradish peroxidase (HRP)-conjugated AffiniPure goat anti-mouse IgG and anti-rabbit IgG were purchased from Zhongshan Golden Bridge Biotechnology Co., Ltd. (Beijing, China).

AS-IV was purchased from Nanjing Zelang Medical Technology Co., Ltd. (Nanjing, China; purity >98\%, HPLC). The chemical structure of AS-IV $\left(\mathrm{C}_{41} \mathrm{H}_{68} \mathrm{O}_{14}\right.$; molecular weight, 784) is depicted in Fig. 1. AS-IV was dissolved in DMSO to the concentration of $25 \mu \mathrm{mol} / \mathrm{ml}$ as a stock solution. The stock solution was diluted with DMEM into AS-IV solutions according to the respective group when used and the final DMSO concentration did not exceed $0.5 \%(\mathrm{v} / \mathrm{v})$.

$M C$ culture. The human mesangial cell line (HMC) was obtained from the Modern Analysis and Testing Center of Central South University (Changsha, China), and maintained in normal DMEM (5.6 mM glucose) supplemented with $10 \% \mathrm{FBS}(\mathrm{v} / \mathrm{v}), 100 \mu / \mathrm{ml}$ penicillin and $100 \mu \mathrm{g} / \mathrm{ml}$ streptomycin at $37^{\circ} \mathrm{C}$ in an atmosphere containing $5 \% \mathrm{CO}_{2} . \mathrm{HG}$ treatment was performed by culturing the cells in DMEM containing $25 \mathrm{mM}$ glucose for $48 \mathrm{~h}$.

Cell proliferation assay. Cells were seeded at a density of $1 \times 10^{4}$ cells/well in 96-well plates. When the cell confluence reached at $70-80 \%$, the growth medium was replaced with DMEM containing $5.6 \mathrm{mM}$ glucose and $0.5 \%$ FBS. After $24 \mathrm{~h}$, the quiescent cells were treated with the indicated concentrations of glucose together with various concentrations of $\operatorname{AS}-\mathrm{IV}(5,10,25,50$ and $100 \mu \mathrm{M})$ or $0.5 \%$ DMSO (vehicle control) for $48 \mathrm{~h}$. The osmotic control medium was made by supplementing normal DMEM with 24.5 mM MA. Following incubation with the above-mentioned compounds, cell proliferation was determined by MTT assay (37). The absorbance was measured at $490 \mathrm{~nm}$ using a SpectraMax 190 Microplate Reader (Molecular Devices, Sunnyvale, CA, USA). 


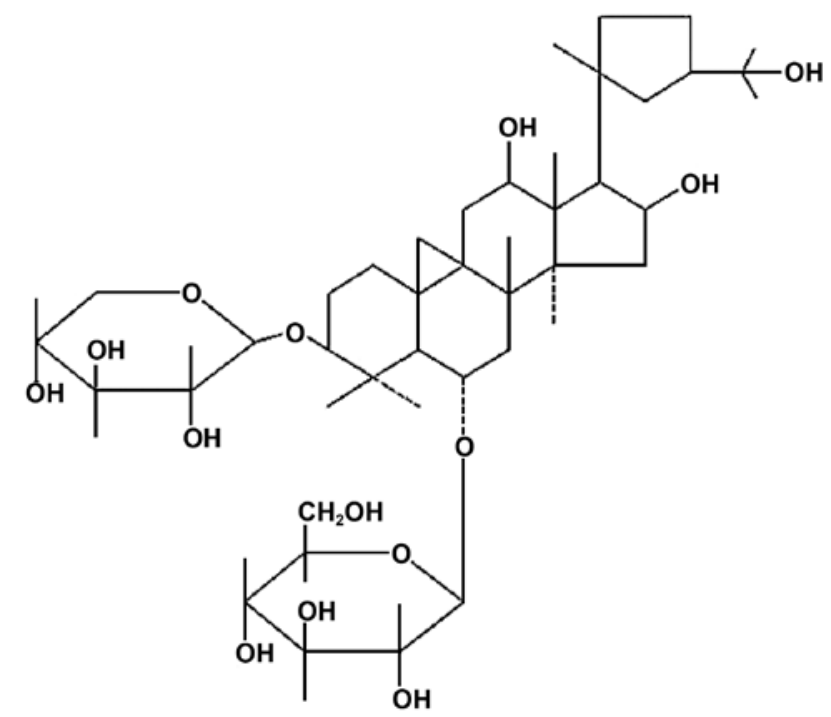

Figure 1. Chemical structure of astragaloside IV (AS-IV).

Measurement of total protein to cell count ratio. The ratio of the total protein content to the cell number is a well-established measure of cellular hypertrophy (38). The MCs were seeded into 6-well plates and were synchronized into quiescence in DMEM containing a normal glucose concentration and $0.5 \%$ FBS for $24 \mathrm{~h}$. The cells were then stimulated with $\mathrm{HG}$ and treated with various concentrations of AS-IV $(5,10,25$, 50 and $100 \mu \mathrm{M}$ ) or $0.5 \%$ DMSO (vehicle control) for $48 \mathrm{~h}$. Following incubation, the cells were washed twice with PBS and trypsinized. A small aliquot of the cells was used for cell counting by a hemocytometer. The remaining cells were lysed in RIPA buffer (Beyotime, Haimen, China), and the total protein content was measured by a protein quantitative reagent kit-BCA method (Beyotime). The total protein content to the cell count ratio was expressed as microgram protein per $10^{4}$ cells.

Detection of intracellular ROS generation. The generation of ROS was detected using the membrane permeable indicator, DCFH-DA. The cells were seeded in 24-well plates at a density of $1 \times 10^{5}$ cells/well. After being synchronized, the cells were cultured in DMEM containing 5.6 or $25 \mathrm{mM}$ glucose with or without various concentrations of AS-IV $(25,50$ and $100 \mu \mathrm{M})$ or tempol $(100 \mu \mathrm{M})$ for $48 \mathrm{~h}$, were then loaded with $10 \mu \mathrm{M}$ DCFH-DA in serum-free DMEM containing 5.6 or $25 \mathrm{mM}$ glucose at $37^{\circ} \mathrm{C}$ for $30 \mathrm{~min}$ in the dark, and the cell culture plate was shaken every $5 \mathrm{~min}$ and washed three times with PBS in order to remove residual probes. Subsequently, intracellular ROS production were observed under a fluorescence microscope (Nikon, Tokyo, Japan; excitation at $488 \mathrm{~nm}$, emission at $525 \mathrm{~nm}$ ). The mean fluorescence intensity for each group of cells was determined using the Image-Pro Plus 6.0 analysis system (MediaCybernetics, Rockville, MD, USA).

NADPH oxidase activity assay. NADPH oxidase activity in the MCs was measured using the cell NADPH oxidase colorimetric assay kit (GenMed Scientifics Inc.) according to the manufacturer's instructions. Briefly, the cells grown in DMEM containing 5.6 or $25 \mathrm{mM}$ glucose in the presence or absence of AS-IV $(25,50$ and $100 \mu \mathrm{M})$ or DPI $(10 \mu \mathrm{M})$ for $48 \mathrm{~h}$ were washed twice in PBS and scraped from the plate followed by centrifugation at $12,000 \mathrm{~g}, 4^{\circ} \mathrm{C}$, for $3 \mathrm{~min}$, and suspended in PBS, followed by incubation with $250 \mu \mathrm{M}$ NADPH. NADPH consumption was monitored by a decrease in absorbance at $340 \mathrm{~nm}$ for $5 \mathrm{~min}$ using a SpectraMax 190 Microplate Reader (Molecular Devices). NADPH oxidase activity was defined as picomoles per liter of substrate per minute per milligram of protein.

Western blot analysis. The cells were lysed with cold lysis buffer containing protease inhibitors. Equal amounts of protein extracts were fractionated by SDS-PAGE and then transferred onto polyvinylidene difluoride (PVDF) membranes (Millipore Corp., Bedford, MA, USA). After being blocked with 5\% non-fat milk in Tris-buffered saline with Tween-20 (TBST, $\mathrm{pH}$ 7.6) for $1 \mathrm{~h}$ at room temperature, the membranes were incubated with the indicated primary antibodies (Nox4, 1:200; Akt, 1:500; phospho-Akt, 1:500;

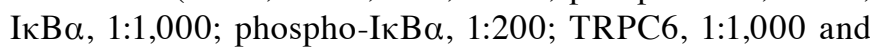
$\beta$-actin, 1:1,000) overnight at $4^{\circ} \mathrm{C}$. The membranes membranes were rinsed three times with TBST and incubated with the respective secondary antibodies (1:10,000 dilutions of each antibody) for $1 \mathrm{~h}$ at room temperature. The protein bands were visualized with SuperSignal West Femto Maximum Sensitivity Substrate (Thermo Fisher Scientific Inc., Rockford, IL, USA) and captured using a Bioshine ChemiQ 4600 Mini Chemiluminescence imaging system (Ouxiang, Shanghai, China). The optical density of each band was quantified using Image J software (NIH, Bethesda, MD, USA) and normalized to the intensity of $\beta$-actin.

Fluorescence measurement of $\left[\mathrm{Ca}^{2+}\right]_{i}$. Measurements of the intracellular free calcium concentration were performed using Fluo-3/AM fluorospectrophotometry. The MCs, grown on 24-well plates, were loaded with Fluo-3 by incubation for $40 \mathrm{~min}$ at $37^{\circ} \mathrm{C}$ in the dark in HEPES buffer solution containing $5 \mu \mathrm{M}$ Fluo-3/AM followed by washing three times with the same buffer. The cells were then incubated with Fluo-3-free HEPES buffer for an additional $20 \mathrm{~min}$. Afer being trypsinized and collected, the fluorescence intensity $(\mathrm{F})$ was monitored using an F-4600 fluorescence spectrophotometer (Hitachi, Tokyo, Japan; excitation at $488 \mathrm{~nm}$, emission at $530 \mathrm{~nm})$. The maximal density $\left(\mathrm{F}_{\text {max }}\right)$ was measured using $0.1 \%$ Triton $\mathrm{X}-100$, and the minimum $\left(\mathrm{F}_{\min }\right)$ was measured using $20 \mathrm{mM}$ EGTA. The concentration of calcium was calculated using the following formula: $\left[\mathrm{Ca}^{2+}\right]_{\mathrm{i}}=\mathrm{K}_{\mathrm{d}}\left(\mathrm{F}-\mathrm{F}_{\min }\right) /\left(\mathrm{F}_{\max }\right.$ - F), where $K_{d}$ represents the dissociation constant of Fluo-3 and calcium, and its value is $450 \mathrm{nM}$.

Statistical analysis. Data are presented as the means \pm standard deviation (SD). Each experiment was repeated at least three times independently. Statistical analysis was performed using SPSS 13.0 for Windows (SPSS, Inc., Chicago, IL, USA). Statistical differences between two groups were analyzed by the unpaired Student's t-test and differences among multiple groups were analyzed by one-way ANOVA. In all cases, values of $\mathrm{P}<0.05$ were considered to indicate statistically significant differences. 

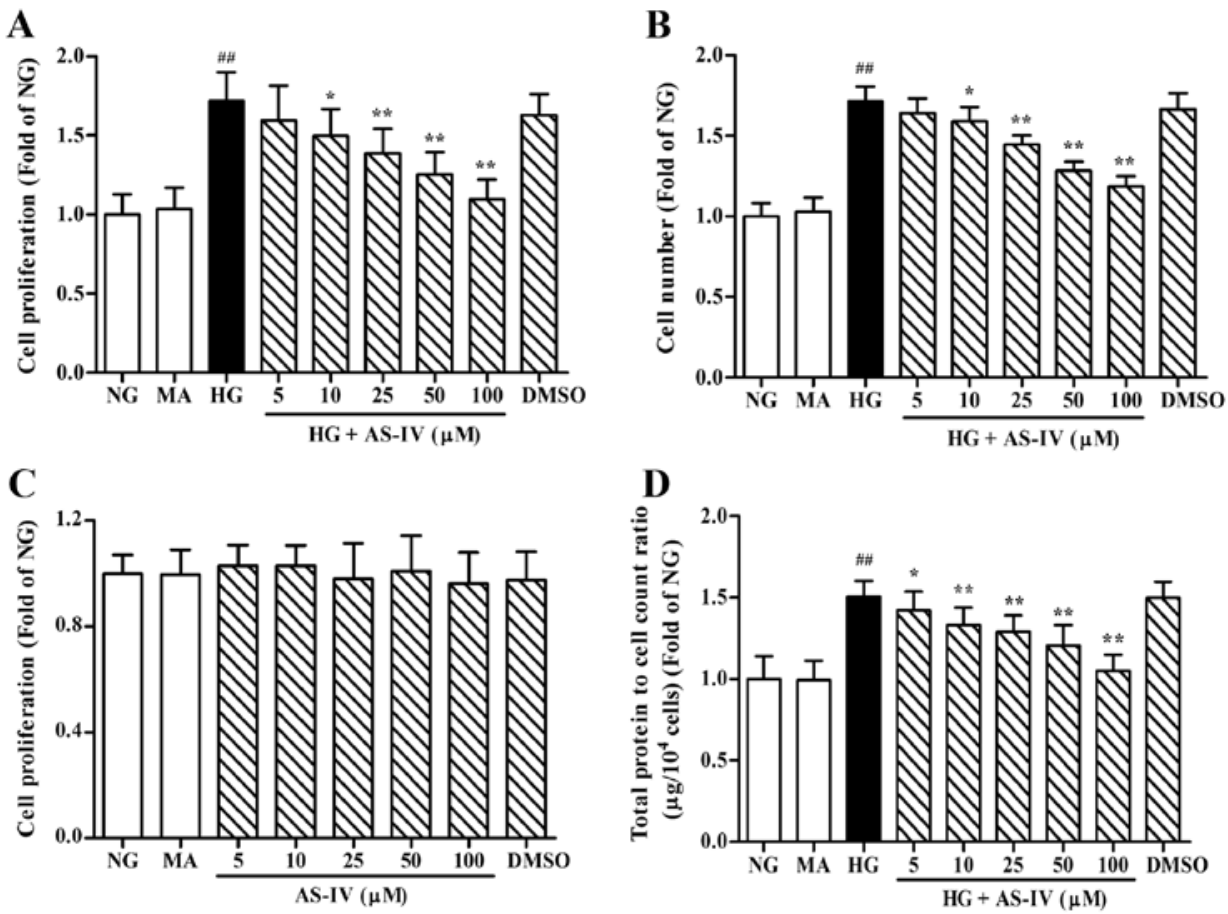

Figure 2. Astragaloside IV (AS-IV) suppresses mesangial cell (MC) proliferation and hypertrophy stimulated by high glucose (HG). (A and B) MCs were cultured in Dulbecco's modified Eagle's medium (DMEM) containing 5.6 [normal glucose (NG)] or $25 \mathrm{mM} \mathrm{HG}$ in the presence or absence of AS-IV (5, 10, 25, 50 and $100 \mu \mathrm{M}$ ) or $25 \mathrm{mM} \alpha$-mannitol (MA) or $0.5 \%$ dimethyl sulfoxide (DMSO) for $48 \mathrm{~h}$. Following incubation, cell proliferation was examined by MTT assay and the cell number was calculated. (C) MCs were treated with NG in the presence or absence of AS-IV (5, 10, 25, 50 and $100 \mu \mathrm{M})$ or $0.5 \%$ DMSO for $48 \mathrm{~h}$. (D) MCs were incubated under HG or NG conditions with or without AS-IV (5-100 $\mu \mathrm{M})$ or $25 \mathrm{mM}$ MA or $0.5 \%$ DMSO for $48 \mathrm{~h}$. Cell hypertrophy was determined with the ratio of total protein content to the cell number which expressed as $\mu \mathrm{g} / 10^{4}$ cells. ${ }^{\# \#} \mathrm{P}<0.01$ compared with cells cultured in NG; ${ }^{*} \mathrm{P}<0.05,{ }^{* *} \mathrm{P}<0.01 \mathrm{compared}$ with cells cultured in HG. Data in the bar graphs represent the means \pm standard deviation (SD) of three independent experiments.

\section{Results}

Effects of AS-IV on HG-induced MC proliferation and hypertrophy. To determine the effects of AS-IV on MC proliferation, the MTT assay and cell counting were employed. As shown in Fig. 2A and B, HG significantly stimulated the growth of the MCs in comparison to normal glucose (NG) conditions $(\mathrm{P}<0.01)$. The administration of AS-IV at the concentration range of 5-100 $\mu \mathrm{M}$ led to a significant inhibition of cell growth induced by HG. The vehicle control treated with DMEM containing HG and 0.5\% DMSO also showed a significant increase in the proliferation of MCs. In addition, AS-IV at the examined concentrations had no effect on the viability of MCs under NG conditions, which suggested that the inhibitory effect of AS-IV upon cultured MCs was not due to its cytotoxicity (Fig. 2C). HG also markedly stimulated cell hypertrophy, defined as the protein content of MCs per unit cell number, which was abolished by the AS-IV administration in a dose dependent manner (Fig. 2D). Unlike HG, the addition of $24.5 \mathrm{mM}$ MA to the medium did not exert an obvious effect on the proliferation and hypertrophy of MCs as compared with control, suggesting that the HG-triggered MC proliferation and hypertrophy were not the results of high osmolality within the medium.

Effect of AS-IV on HG-stimulated ROS generation in MCs. We examined the effects of AS-IV on HG-induced intracellular ROS generation by DCFH-DA fluorescent probe assay using fluorescence microscopy. As demonstrated in Fig. 3, the
MCs cultured under HG conditions for $48 \mathrm{~h}$ showed a significant increase in ROS generation compared to the cells cultured under NG conditions $(\mathrm{P}<0.01)$. To assess the antioxidative effects of AS-IV, we used tempol, a classic antioxidant, as a positive control. The effects of HG-induced ROS generation in the MCs were notably decreased by treatment with AS-IV (25, 50 and $100 \mu \mathrm{M})$ or tempol $(100 \mu \mathrm{M})$, and AS-IV exerted its antioxidant effects in a dose-dependent manner.

\section{Effect of AS-IV on HG-mediated NADPH oxidase activation} in MCs. Since NADPH oxidase is the most important source of intracellular ROS, we used the NADPH oxidase inhibitor, DPI, as a positive control to assess the effects of AS-IV on HG-induced NADPH oxidase activity. As shown in Fig. 4A, HG resulted in a significant increase in NADPH activity that was markedly attenuated by AS-IV $(25,50$ and $100 \mu \mathrm{M})$ or DPI $(10 \mu \mathrm{M})$. As Nox 4 is the key membrane subunit of NADPH oxidase expressed in MCs, we further examined whether AS-IV blocks the expression level of Nox 4 protein. The protein level of the Nox4 subunit was notably upregulated under HG conditions compared to the control $(\mathrm{P}<0.01)$. Treatment with AS-IV $(25,50$ and $100 \mu \mathrm{M})$ or DPI $(10 \mu \mathrm{M})$ for $48 \mathrm{~h}$ markedly downregulated the protein expression level of Nox4 compared to the HG-treated group (Fig. 4B and C).

Inhibitory effects of AS-IV on HG-induced phosphorylation of $A k t$ and I $K B \alpha$ in MCs. Given the role that Akt and $\mathrm{NF}-\mathrm{\kappa B}$ signaling plays in MC growth and proliferation, we examined the effects of AS-IV on Akt and NF- $\mathrm{KB}$ activation. 

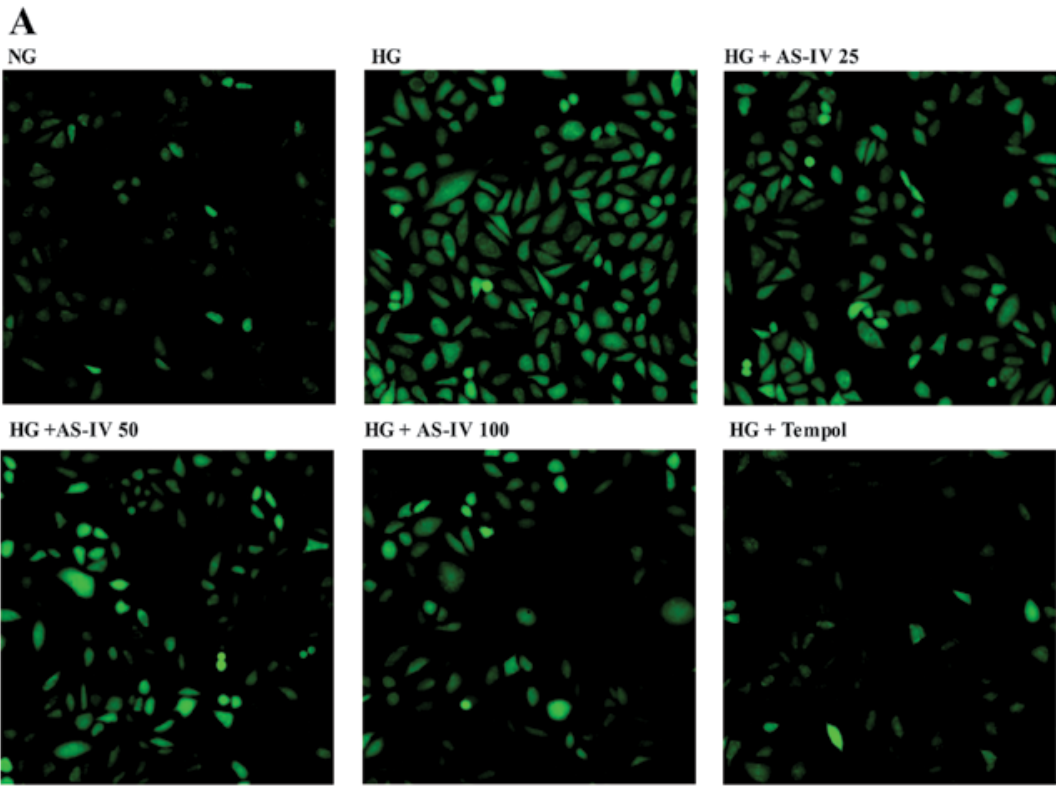

HG + AS-IV 100
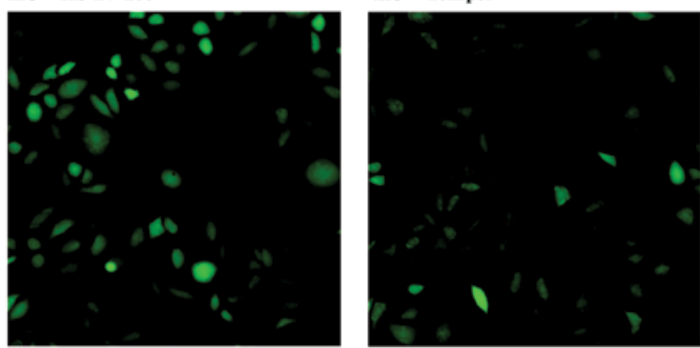

B

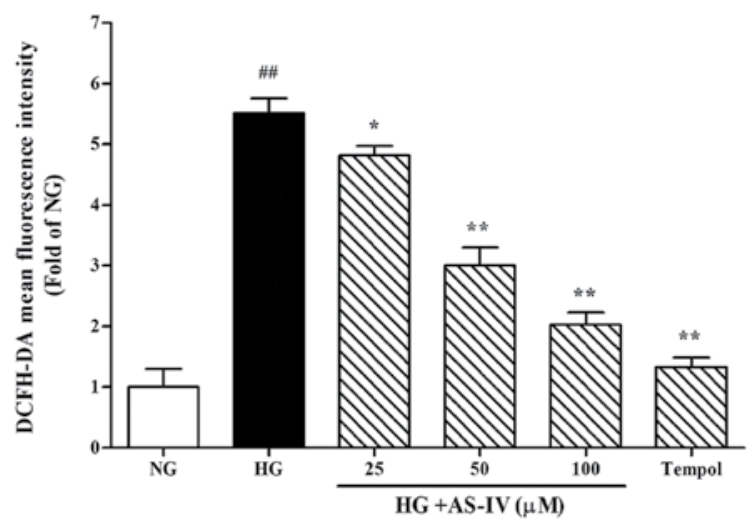

Figure 3. Astragaloside IV (AS-IV) inhibits high glucose (HG)-induced reactive oxygen species (ROS) generation in mesangial cells (MCs). (A) MCs were treated with normal glucose (NG) or HG in the presence or absence of AS-IV (25, 50 and $100 \mu \mathrm{M})$ or tempol $(100 \mu \mathrm{M})$ for 48 h. Cells were stained with the ROS-sensitive dye fluorescent probe, 2',7'-dichlorofluorescein diacetate (DCFH-DA), and observed under a fluorescence microscope. Magnification, x200. (B) The average fluorescence intensity of the NG group was calculated as $100 \%$, and all data were normalized to the NG group. ${ }^{\# \#} \mathrm{P}<0.01$ compared with NG; ${ }^{*} \mathrm{P}<0.05,{ }^{* *} \mathrm{P}<0.01$ compared with HG. Data in the bar graphs represent the means \pm standard deviation (SD) of three independent experiments.

A

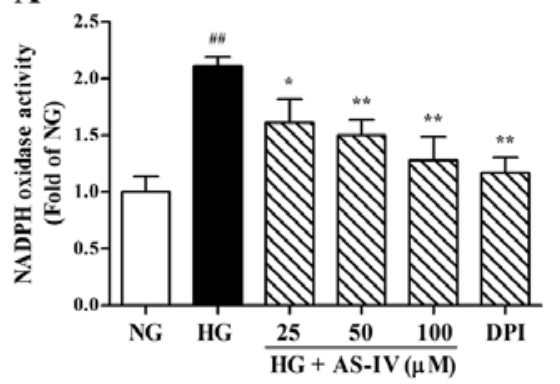

B

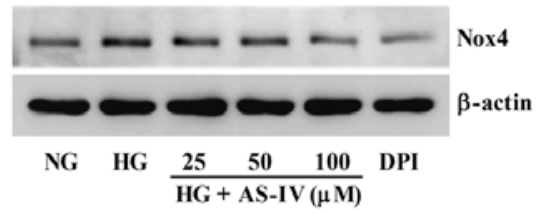

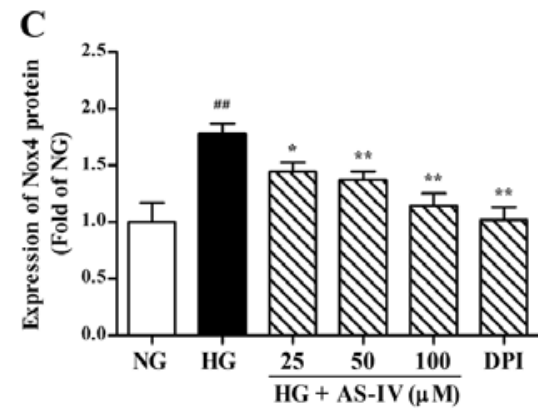

Figure 4. High glucose (HG)-mediated nicotinamide adenine dinucleotide phosphate (NADPH) oxidase activation was inhibited by astragaloside IV (AS-IV) in mesangial cells (MCs). (A) MCs were incubated with normal glucose (NG) or HG for $48 \mathrm{~h}$ and with or without AS-IV (25, 50 and $100 \mu \mathrm{M})$ or diphenyleneiodonium (DPI) $(10 \mu \mathrm{M})$. The activity of NADPH oxidase was detected using the cell NADPH oxidase colorimetric assay kit. (B) The protein level of Nox4 after HG treatment for $48 \mathrm{~h}$ was determined by western blot analysis, normalized to $\beta$-actin. (C) Summary data from the experiments indicated in (B). The Nox $4 / \beta$-actin ratios were expressed relative to the mean value of the control. ${ }^{\# \prime} \mathrm{P}<0.01$ compared with $\mathrm{NG}$; $\mathrm{P}<0.05,{ }^{* * *} \mathrm{P}<0.01$ compared with HG. Values are the means \pm standard deviation (SD) of three independent experiments.

The phosphorylation level of Akt and IкB $\alpha$ and the protein expression of total Akt and total $\mathrm{I} \kappa \mathrm{B} \alpha$ were detected by western blot analysis. It was observed that $\mathrm{HG}$ induced Akt and NF- $\kappa B$ activation, as manifested by the fact that the 
A

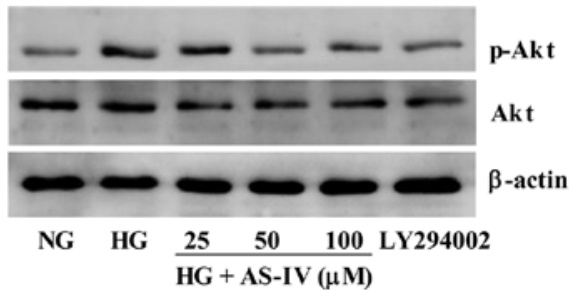

C

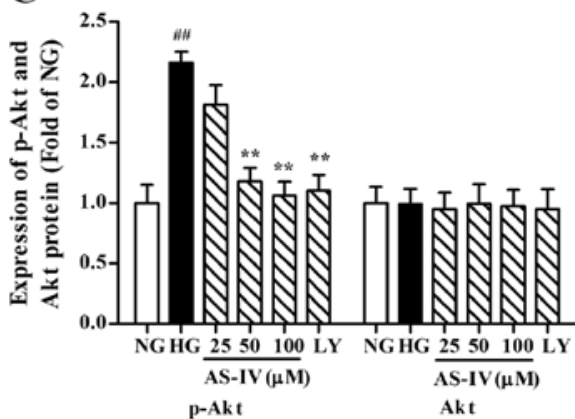

B

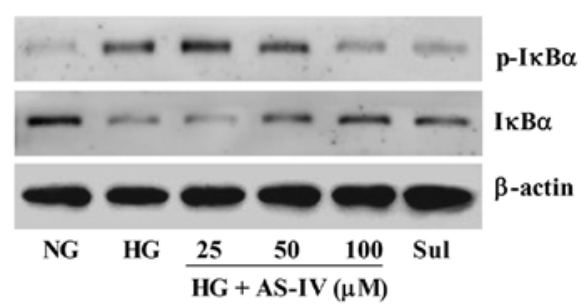

D

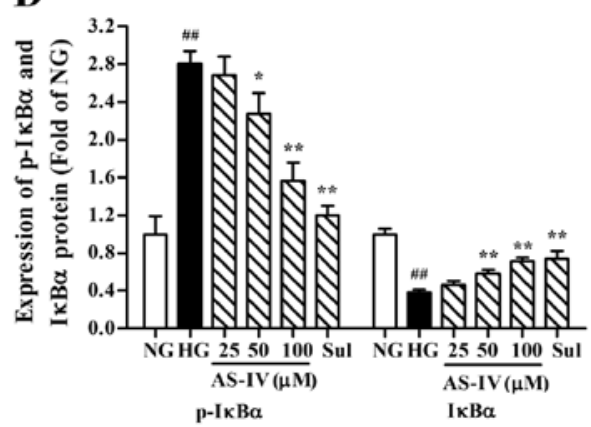

Figure 5. Astragaloside IV (AS-IV) suppresses high glucose (HG)-induced Akt and nuclear factor- $\kappa \mathrm{B}$ (NF- $\mathrm{B}$ ) activation in mesangial cells (MCs). (A) MCs were cultured in normal glucose (NG) or HG with or without various concentrations of AS-IV (25, 50 and $100 \mu \mathrm{M})$ or LY294002 (10 $\mu \mathrm{M})$ for $48 \mathrm{~h}$. Akt phosphorylation and total Akt protein level was detected by western blot analysis. (B) MCs were cultured in NG or HG with or without various concentration

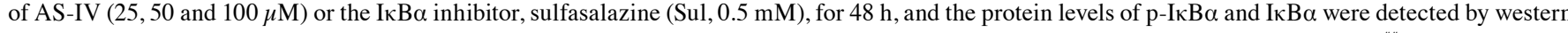

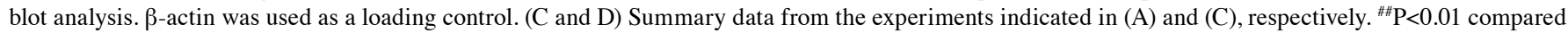
with NG; ${ }^{*} \mathrm{P}<0.05,{ }^{* *} \mathrm{P}<0.01$ compared with HG. Values are the means \pm standard deviation (SD) of three independent experiments.

relative amount of phosphorylated Akt and $\mathrm{I} \kappa \mathrm{B} \alpha$ and the degradation of I $\kappa \mathrm{B} \alpha$ were significantly higher compared to the control cells (Fig. 5). However, treatment with AS-IV or the PI3K inhibitor, LY294002 $(10 \mu \mathrm{M})$, effectively abrogated the HG-induced Akt phosphorylation in the MCs, and the phosphorylation and degradation level of I $\mathrm{B} \alpha$ was also markedly inhibited by AS-IV or the I $\mathrm{B} \alpha$ inhibitor, Sul (0.5 mM). AS-IV did not affect the protein expression level of total Akt in the HG-cultured cells. These results suggest that AS-IV inhibits $\mathrm{HG}$-induced Akt and NF- $\kappa \mathrm{B}$ activation in MCs.

Regulatory effect of AS-IV on the NADPH oxidase/ROS/Akt/ $N F-\kappa B$ signaling pathway in MCs under $H G$ conditions. To further investigate the signaling cascade involved in HG-induced NADPH oxidation, Akt and NF- $\kappa$ B activation, specific inhibitors of several signaling molecules were used. Treatment with NADPH oxidase inhibitor (DPI, $10 \mu \mathrm{M}$ ) or ROS inhibitor (tempol, $100 \mu \mathrm{M}$ ) blocked Akt and NF- $\kappa \mathrm{B}$ activation in the MCs induced by HG (Fig. 6), indicating that NADPH oxidase activation induced by HG occurs upstream of Akt and NF- $\kappa \mathrm{B}$ in MCs. The HG-induced Akt phosphorylation was abolished by treatment with PI3K inhibitor (LY294002, $10 \mu \mathrm{M})$, whereas Sul $(0.5 \mathrm{mM})$, an I $\mathrm{B} \mathrm{B} \alpha$ inhibitor, failed to inhibit Akt phosphorylation (Fig. 6A and C). I $\kappa \mathrm{B} \alpha$ phosphorylation and degradation were also inhibited in the presence of Sul, LY294002 and DPI or tempol (Fig. 6B and D). These data demonstrate that the PI3K/Akt pathway is upstream of $\mathrm{NF}-\kappa \mathrm{B}$ in MCs cultured under hyperglycemic conditions.

We also examined whether NADPH oxidase activation in $\mathrm{MCs}$ is dependent on Akt or $\mathrm{NF}-\kappa \mathrm{B}$ activation. The expression level of the Nox4 subunit was evaluated in the HG-exposed cells treated with or without LY294002, DPI, tempol or Sul. LY294002 and DPI effectively inhibited the HG-induced increase in the expression of Nox4. However, no significant change in the expression level of Nox4 was observed in the MCs treated with tempol and Sul (Fig. 6E and F). Taken together, the above results suggest that NADPH oxidase activation and the PI3K/Akt pathway may function in parallel or may interplay with each other, which are upstream of $\mathrm{NF}-\kappa \mathrm{B}$ in $\mathrm{HG}$-stimulated MCs.

Inhibitory effects of AS-IV on the HG-induced downregulation of TRPC6 and the reduction in calcium influx in MCs. TRPC6 is known as a $\mathrm{Ca}^{2+}$-conductive cation channel and regulates the contractile function of MCs; it plays a pivotal role during the early stages of HG-induced damage to MCs $(14,15)$. Thus, in this study, we examined the effects of AS-IV on the expression level of TRPC6 protein and the concentration of intracellular free calcium in MCs cultured under HG conditions. As illustrated in Fig. 7A and C, incubation of the MCs with HG for $48 \mathrm{~h}$ markedly decreased the expression level of TRPC6 protein compared with the cells cultured under NG conditions. The downregulation of TRPC6 induced by HG was markedly abrogated by treatment with AS-IV at a concentration of 25 to $100 \mu \mathrm{M}$ and the TRPC6 agonist, a diacylglycerol analog, 1-oleoyl-2-acetyl-sn-glycerol (OAG, $100 \mu \mathrm{M})$. Furthermore, in the presence of inhibitors of signaling molecules, such as LY294002, DPI, tempol and Sul, the HG-induced TRPC6 downregulation was markedly abolished (Fig. 7B and D). Ultimately, we detected the intracellular free calcium concentration in MCs using Fluo-3/AM fluorospectrophotometry. As shown in Fig. 7E, the HG-induced reduction in calcium influx in the MCs was also greatly ameliorated by AS-IV (25, 50 and $100 \mu \mathrm{M})$ or OAG $(100 \mu \mathrm{M})$. These results suggest that AS-IV protects MCs against contractile dysfunction under HG conditions by upregulating the TRPC6 protein 
A

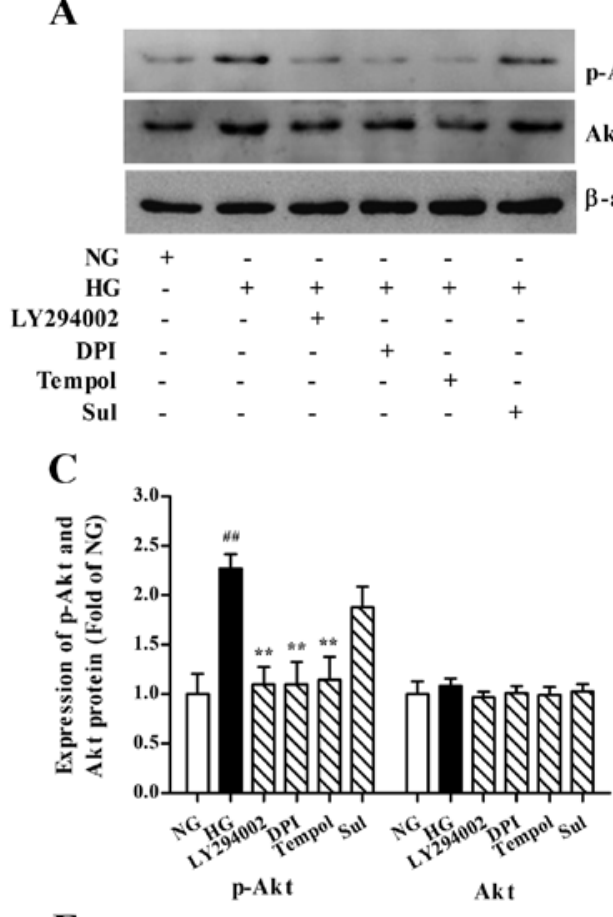

B

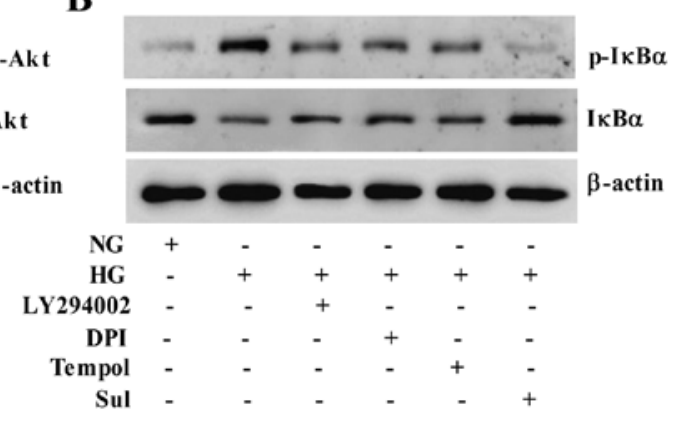

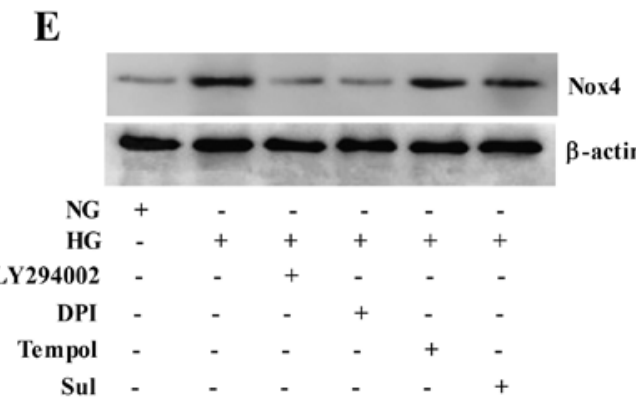
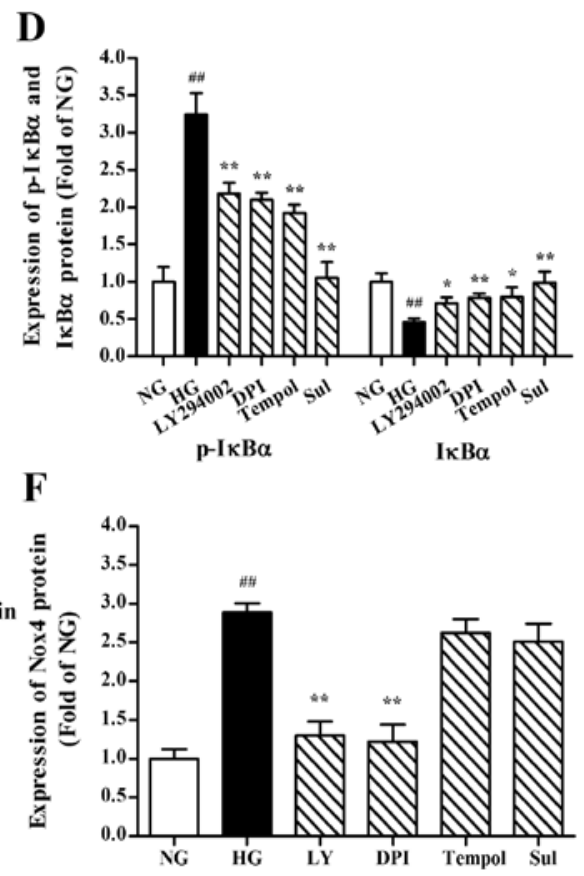

Figure 6. High glucose (HG)-potentiated nicotinamide adenine dinucleotide phosphate (NADPH) oxidase activation is affected by the phosphoinositide 3-kinase (PI3K)/Akt pathway, but not nuclear factor- $\mathrm{kB}(\mathrm{NF}-\kappa \mathrm{B})$ in mesangial cells (MCs). (A, B and E) Cells were incubated with HG in presence or absence of various inhibitors [LY294002, $10 \mu \mathrm{M}$; diphenyleneiodonium (DPI), $10 \mu \mathrm{M}$; tempol, $100 \mu \mathrm{M}$; sulfasalazine (Sul), $0.5 \mathrm{mM})$ ] for $48 \mathrm{~h}$. The

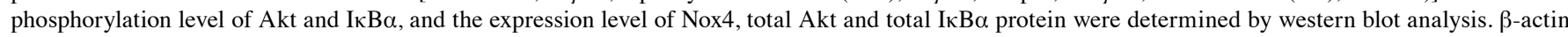
was used as a loading control. (C, D and F) Representative quantitative analysis for results of (C) Akt, (D) IkB $\alpha$, and (F) Nox4 were expressed as fold change from the control. ${ }^{\# \#} \mathrm{P}<0.01$ compared with normal glucose (NG); ${ }^{*} \mathrm{P}<0.05,{ }^{* *} \mathrm{P}<0.01$ compared with HG. Data in the bar graphs represent the means \pm standard deviation (SD) of three independent experiments.

expression and increasing $\mathrm{Ca}^{2+}$ influx through the $\mathrm{NADPH}$ oxidase/ROS/Akt/NF- $\mathrm{\kappa B}$ signaling pathway.

\section{Discussion}

Radix Astragali, the dried root of Astragalus membranaceus (Fisch.) Bunge, has long been used in traditional Chinese medicine for the treatment of cardiovascular diseases and diabetes (39). Recently, investigations into its active ingredients have attracted much attention due to the unique pharmacological properties of many of its constituents (34). AS-IV is a novel saponin extracted from Radix Astragali, and it has been reported to ameliorate podocyte apoptosis by attenuating ROS production and to prevent acute kidney injury by inhibiting oxidative stress $(40,41)$. In a previous study of ours, we suggested that AS-IV significantly reduced $\mathrm{H}_{2} \mathrm{O}_{2}$ induced ROS overproduction in MCs (42). In order to further demonstrate that treatment with AS-IV can suppress oxidative stress-induced injury in DN, the present study was designed to examine the protective effects of AS-IV on the morphological and functional abnormalities of MCs cultured under hyperglycemic conditions.

Hyperglycemia, a common condition occurring in diabetes, markedly increases the production of ROS in MCs (19). The redox imbalance between the production of ROS and the compensatory response from the endogenous antioxidant network results in oxidative stress. The interaction of the excessive ROS generation with biomolecules, such as lipids, proteins and DNA, can activate a series of cell signaling pathways, leading to severe kidney injury and dysfunction (43). The most prominent effect is MC proliferation, which often leads to glomerulosclerosis (GS), renal fibrosis or even end-stage renal failure (44). NADPH oxidase, a multicomponent enzyme, is the major source of ROS production in renal cells (23). NADPH oxidase was originally found in neutrophils (22). In many non-phagocytic cells, the Nox family is a homologue of gp $91^{\text {phox }}$, which is the catalytic subunit of NADPH oxidase, including several types, 
A

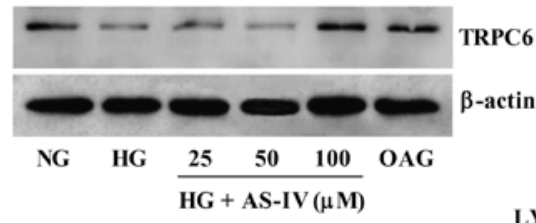

B

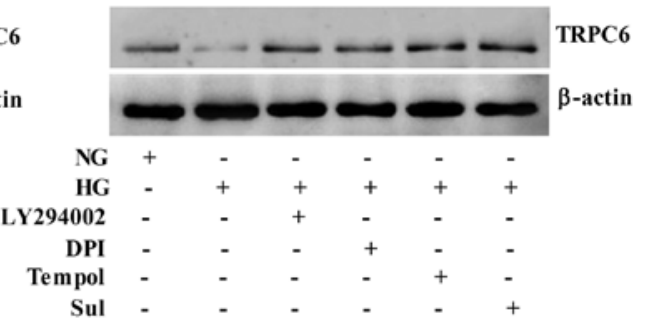

D

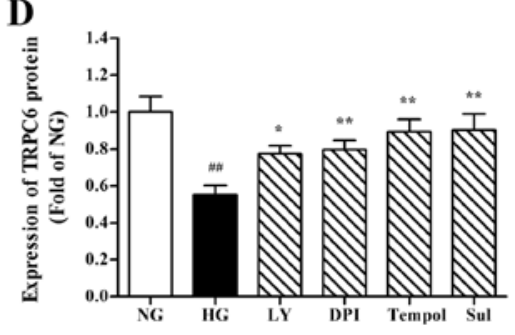

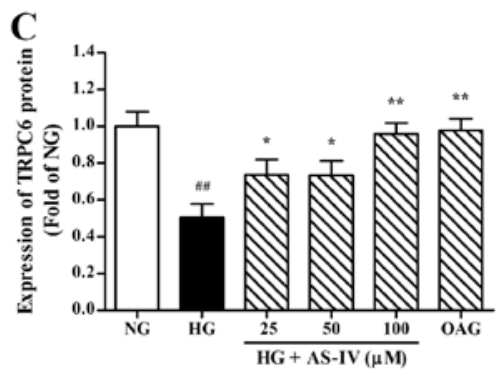

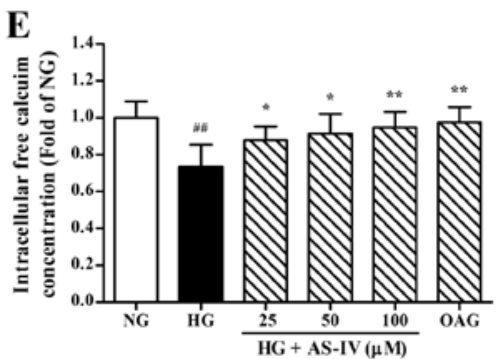

Figure 7. Treatment with astragaloside IV (AS-IV) inhibits the downregulation of canonical transient receptor potential cation channel 6 (TRPC6) protein in mesangial cells (MCs) and the reduction in calcium influx under high glucose (HG) conditions. (A) MCs were stimulated with HG for $48 \mathrm{~h}$ with or without AS-IV at various concentration $(25,50$, and $100 \mu \mathrm{M})$ or 1-oleoyl-2-acetyl-sn-glycerol (OAG) (100 $\mu \mathrm{M})$. (B) MCs were cultured in normal glucose (NG) or HG with or without different inhibitors of signaling molecules [LY294002, $10 \mu \mathrm{M}$; diphenyleneiodonium (DPI), $10 \mu \mathrm{M}$; tempol, $100 \mu \mathrm{M}$; sulfasalazine (Sul), 0.5 $\mathrm{mM}]$ for $48 \mathrm{~h}$. The protein level of TRPC6 was detected by western blot analysis, and $\beta$-actin was used as a loading control. (C and D) Summary data from the experiments indicated in (A) and (B), respectively. (E) MCs were loaded with the fluoroprobe Fluo-3/AM, and the fluorescence intensity of intracellular calcium was detected by fluorospectrophotometry. The $\mathrm{Ca}^{2+}$ concentration was expressed as fold of NG. ${ }^{\# \#} \mathrm{P}<0.01$ compared with $\mathrm{NG}$; ${ }^{*} \mathrm{P}<0.05$, ${ }^{* *} \mathrm{P}<0.01$ compared with HG. Results represent the means \pm standard deviation (SD) of three independent experiments.

such as Nox1, Nox2, Nox3, Nox4 and Nox5 (45). Of these, the Nox4 isoform is mainly found in MCs (46). The activity of NADPH oxidase and the expression level of Nox4 protein are both markedly increased in MCs under HG conditions. In this study, we found that treatment with AS-IV markedly supressed HG-induced intracellular ROS generation, as well as MC proliferation and hypertrophy. Consistently, our experiments demonstrated that AS-IV markedly attenuated the HG-stimulated NADPH oxidase activation and the overexpression of Nox4 in MCs. Our results indicated that there was no obvious direct cytotoxic effect of AS-IV on MCs. These results provide evidence that AS-IV may exert an inhibitory effect on HG-induced MC proliferation and hypertrophy by downregulating Nox4-derived ROS generation.

Since the activation of various cellular molecules, such as transcription factors, cytokines, hormones and protein kinases has been reported to contribute to the signal transduction cascades of DN $(47,48)$, we investigated the mechanisms through which AS-IV prevents damage to human MCs induced by HG stimulation in detail in order to explore the underlying molecular mechanisms involved in the above-mentioned effects of AS-IV. Our results revealed that following HG stimulation, the levels of several cellular phosphorylated molecules were decreased by treatment with AS-IV. Akt, one of the downstream effectors of PI3K, is involved in cell proliferation and hypertrophy (26). It has been demonstrated that Akt is activated in renal cells and is regulated by intracellular ROS (28). In the present study, we found that the phosphorylation level of Akt was increased by HG stimulation in MCs. Akt phosphorylation was also markedly reduced by treatment with AS-IV. Moreover, Akt plays a key role in promoting the transcriptional activity of $N F-\kappa B(29)$. NF- $\kappa \mathrm{B}$ is one of the most important transcription factors, which can be activated by various stimuli in DN, such as hyperglycemia, advanced glycation end products (AGEs), angiogeninII (AngII), oxidative stress and the PI3K/Akt signaling pathway (49). Akt promotes the activation of NF- $\kappa \mathrm{B}$ by activating the I $\kappa \mathrm{B}$ kinase (IKK) to accelerate the phosphorylation and degradation of $\mathrm{I} \kappa \mathrm{B}$, thereby promoting the translocation of $\mathrm{NF}-\kappa \mathrm{B}$ from the cytoplasm into the nucleus and subsequently binding to specific sequences in DNA, which in turn results in gene transcription. These signaling cascades eventually lead to MC proliferation (31). Our results revealed that $\mathrm{HG}$ enhanced the activation of $\mathrm{NF}-\kappa \mathrm{B}$, and that the degradation and phosphorylation levels of $\mathrm{I} \kappa \mathrm{B} \alpha$ were marekdly 
decreased in the MCs treated with AS-IV. These data strongly suggest that the Akt/NF- $\kappa \mathrm{B}$ signaling pathway is involved in the pathogenesis of $\mathrm{DN}$.

In addition, we used specific inhibitors of signaling molecules as a control to compare the effectiveness of AS-IV in order to validate the upstream and downstream association among $\mathrm{HG}$-induced NADPH oxidase, Akt and NF- $\kappa \mathrm{B}$ activation. The phosphorylation levels of Akt and $\mathrm{I} \kappa \mathrm{B} \alpha$ were markedly inhibited by the NADPH oxidase inhibitor, DPI, or the ROS inhibitor, tempol. Of note, the PI3K inhibitor, LY294002, also abolished the HG-stimulated Nox4 expression, as well as I $\mathrm{B} \alpha$ phosphorylation and degradation. However, the I $\mathrm{B} \alpha$ inhibitor, Sul, did not suppress Nox4 expression and Akt activation induced by HG. Studies have indicated that ROS, as an important stimulator of $N F-\kappa B$ activation, mediate the activation of Akt in MCs and other cultured cells (50). However, controversially, there are also data reporting that the intracellular ROS level is regulated by Akt (26). These discrepancies suggest the existence of a cross-talk between NADPH oxidase-derived ROS and Akt activation. Our results support this assumption and indicate that both the activation of NADPH oxidase and Akt may be required for $\mathrm{HG}$-induced $\mathrm{I} \kappa \mathrm{B} \alpha$ phosphorylation and degradation in MCs.

The early distinctive pathological characteristics of DN are not only MC proliferation, but also the hypocontractility of MCs, which is induced by the decreased $\mathrm{Ca}^{2+}$ influx. TRPC6 is $\mathrm{Ca}^{2+}$ permeable cation channel which plays a pivotal role in regulating $\mathrm{Ca}^{2+}$ signaling in MCs, proving a mechanism for impaired MC contraction in diabetes (10). It has previously been suggested that the abundance of TRPC6 protein in MCs is decreased by ROS and PKC in diabetes (51). Moreover, it has also been reported that $\mathrm{NF}-\kappa \mathrm{B}$ participates in the regulation of TRPC6 expression (33). The results of the present study revealed that exposure to $\mathrm{HG}$ resulted in the downregulation of TRPC6 protein and a reduction in free calcium concentration in the MCs, inducing the contractile dysfunction of MCs; the NADPH oxidase/ROS/Akt/NF- $\kappa \mathrm{B}$ signaling pathway may also be involved in these effects, which were markedly supressed by treatment with AS-IV.

In conclusion, the present study indicates that hyperglycemia induces glomerular MC proliferation and the downregulation of TRPC6 protein by promoting Nox4 upregulation, ROS generation, Akt and NF- $\kappa$ B activation. Treatment with AS-IV inhibits HG-induced MC proliferation and contractile dysfunction through the NADPH oxidase/ROS/Akt/NF- $\mathrm{B}$ signaling pathway. Therefore, we suggest that AS-IV may be a valuable candidate for the prevention and treatment of early DN. However, other relevant mechanisms underlying the effects of AS-IV require further investigation.

\section{Acknowledgements}

This study was supported by grants from the National Natural Science Foundation of China (no. 81173624), the Nature Science Foundation of Anhui Province (no. 11040606M201) and International scientific and Technological Cooperative Project of Anhui province (no. 1230603007). The authors would like to thank Li Gui and Dake Huang from the Synthetic Laboratory of Anhui Medical University for their helpful technical assistance.

\section{References}

1. Parving HH: Diabetic nephropathy: prevention and treatment. Kidney Int 60: 2041-2055, 2001.

2. Bank N: Mechanisms of diabetic hyperfiltration. Kidney Int 40: 792-807, 1991.

3. Wei P, Lane PH, Lane JT, Padanilam BJ and Sansom SC: Glomerular structural and functional changes in a high-fat diet mouse model of early-stage Type 2 diabetes. Diabetologia 47: 1541-1549, 2004.

4. Schlöndorff D and Banas B: The mesangial cell revisited: no cell is an island. J Am Soc Nephrol 20: 1179-1187, 2009.

5. Menè P, Pugliese G, Pricci F, Di Mario U, Cinotti GA and Pugliese F: High glucose level inhibits capacitative $\mathrm{Ca}^{2+}$ influx in cultured rat mesangial cells by a protein kinase C-dependent mechanism. Diabetologia 40: 521-527, 1997.

6. Nutt LK and O'Neil RG: Effect of elevated glucose on endothelin-induced store-operated and non-store-operated calcium influx in renal mesangial cells. J Am Soc Nephrol 11: $1225-1235,2000$

7. Whiteside CI, Hurst RD and Stevanovic ZS: Calcium signaling and contractile response of diabetic glomerular mesangial cells. Kidney Int 51 (Suppl): S28-S33, 1995.

8. Wu JP, Zhang W, Wu F, et al: Honokiol: an effective inhibitor of high-glucose-induced upregulation of inflammatory cytokine production in human renal mesangial cells. Inflamm Res 59: 1073-1079, 2010.

9. Hsu YJ, Hoenderop JG and Bindels RJ: TRP channels in kidney disease. Biochim Biophys Acta 1772: 928-936, 2007.

10. Smyth JT, Hwang SY, Tomita T, DeHaven WI, Mercer JC and Putney JW: Activation and regulation of store-operated calcium entry. J Cell Mol Med 14: 2337-2349, 2010.

11. Nilius B and Owsianik G: The transient receptor potential family of ion channels. Genome Biol 12: 218, 2011

12. Dietrich A and Gudermann T: Trpc6. Handb Exp Pharmacol: 125-141, 2007.

13. Ma R, Du J, Sours S and Ding M: Store-operated $\mathrm{Ca}^{2+}$ channel in renal microcirculation and glomeruli. Exp Biol Med (Maywood) 231: 145-153, 2006.

14. Möller CC, Wei C, Altintas MM, et al: Induction of TRPC6 channel in acquired forms of proteinuric kidney disease. J Am Soc Nephrol 18: 29-36, 2007.

15. Graham S, Ding M, Sours-Brothers S, Yorio T, Ma JX and Ma R: Downregulation of TRPC6 protein expression by high glucose, a possible mechanism for the impaired $\mathrm{Ca}^{2+}$ signaling in glomerular mesangial cells in diabetes. Am J Physiol Renal Physiol 293: F1381-F1390, 2007.

16. Danesh FR, Sadeghi MM, Amro N, et al: 3-Hydroxy-3methylglutaryl CoA reductase inhibitors prevent high glucose-induced proliferation of mesangial cells via modulation of Rho GTPase/p21 signaling pathway: Implications for diabetic nephropathy. Proc Natl Acad Sci USA 99: 8301-8305, 2002.

17. Banday AA, Fazili FR and Lokhandwala MF: Oxidative stress causes renal dopamine D1 receptor dysfunction and hypertension via mechanisms that involve nuclear factor-kappaB and protein kinase C. J Am Soc Nephrol 18: 1446-1457, 2007.

18. Forbes JM, Coughlan MT and Cooper ME: Oxidative stress as a major culprit in kidney disease in diabetes. Diabetes 57: 1446-1454, 2008

19. $\mathrm{Ha} \mathrm{H}$ and Lee HB: Reactive oxygen species as glucose signaling molecules in mesangial cells cultured under high glucose. Kidney Int Suppl 77: S19-S25, 2000.

20. Hool LC and Corry B: Redox control of calcium channels: from mechanisms to therapeutic opportunities. Antioxid Redox Signal 9: 409-435, 2007.

21. Gill PS and Wilcox CS: NADPH oxidases in the kidney. Antioxid Redox Signal 8: 1597-1607, 2006.

22. Babior BM, Lambeth JD and Nauseef W: The neutrophil NADPH oxidase. Arch Biochem Biophys 397: 342-344, 2002.

23. Nauseef WM: Biological roles for the NOX family NADPH oxidases. J Biol Chem 283: 16961-16965, 2008

24. Block K, Eid A, Griendling KK, Lee DY, Wittrant Y and Gorin Y: Nox4 NAD(P)H oxidase mediates Src-dependent tyrosine phosphorylation of PDK-1 in response to angiotensin II: role in mesangial cell hypertrophy and fibronectin expression. J Biol Chem 283: 24061-24076, 2008.

25. Koyasu S: The role of PI3K in immune cells. Nat Immunol 4: 313-319, 2003.

26. Shiojima I and Walsh K: Role of Akt signaling in vascular homeostasis and angiogenesis. Circ Res 90: 1243-1250, 2002 
27. Kim SY, Park KH, Gul R, Jang KY and Kim UH: Role of kidney ADP-ribosyl cyclase in diabetic nephropathy. Am J Physiol Renal Physiol 296: F291-F297, 2009.

28. Kim SY, Gul R, Rah SY, et al: Molecular mechanism of ADP-ribosyl cyclase activation in angiotensin II signaling in murine mesangial cells. Am J Physiol Renal Physiol 294: F982-F989, 2008.

29. Sousa LP, Carmo AF, Rezende BM, et al: Cyclic AMP enhances resolution of allergic pleurisy by promoting inflammatory cell apoptosis via inhibition of PI3K/Akt and NF-kappaB. Biochem Pharmacol 78: 396-405, 2009.

30. Li J and Bertram JF: Review: Endothelial-myofibroblast transition, a new player in diabetic renal fibrosis. Nephrology (Carlton) 15: 507-512, 2010.

31. Yang WS, Seo JW, Han NJ, et al: High glucose-induced NF-kappaB activation occurs via tyrosine phosphorylation of IkappaBalpha in human glomerular endothelial cells: involvement of Syk tyrosine kinase. Am J Physiol Renal Physiol 294: F1065-F1075, 2008.

32. Nitti M, Furfaro AL, Patriarca S, et al: Human mesangial cells resist glycoxidative stress through an antioxidant response. Int J Mol Med 27: 213-219, 2011.

33. Wang XW, Tan BZ, Sun M, Ho B and Ding JL: Thioredoxin-like 6 protects retinal cell line from photooxidative damage by upregulating NF-kappaB activity. Free Radic Biol Med 45: 336-344, 2008.

34. Ren S, Zhang H, Mu Y, Sun M and Liu P: Pharmacological effects of Astragaloside IV: a literature review. J Tradit Chin Med 33: 413-416, 2013.

35. You H, Lu Y, Gui D, Peng A, Chen J and Gu Y: Aqueous extract of Astragali Radix ameliorates proteinuria in adriamycin nephropathy rats through inhibition of oxidative stress and endothelial nitric oxide synthase. J Ethnopharmacol 134: 176-182, 2011.

36. Motomura K, Fujiwara Y, Kiyota N, et al: Astragalosides isolated from the root of astragalus radix inhibit the formation of advanced glycation end products. J Agric Food Chem 57: 7666-7672, 2009.

37. Wang O, Cai K, Pang S, et al: Mechanisms of glucose-induced expression of pancreatic-derived factor in pancreatic beta-cells. Endocrinology 149: 672-680, 2008.

38. Jeong SI, Kim SJ, Kwon TH, Yu KY and Kim SY: Schizandrin prevents damage of murine mesangial cells via blocking NADPH oxidase-induced ROS signaling in high glucose. Food Chem Toxicol 50: 1045-1053, 2012.

39. Wu F and Chen X: A review of pharmacological study on Astragalus membranaceus (Fisch.) Bge. Zhong Yao Cai 27: 232-234, 2004 (In Chinese).
40. Gui D, Guo Y, Wang F, et al: Astragaloside IV, a novel antioxidant, prevents glucose-induced podocyte apoptosis in vitro and in vivo. PloS one 7: e39824, 2012.

41. Gui D, Huang J, Liu W, Guo Y, Xiao W and Wang N: Astragaloside IV prevents acute kidney injury in two rodent models by inhibiting oxidative stress and apoptosis pathways. Apoptosis 18: 409-422, 2013.

42. Cao LL, Li WZ, Si XL, Sun L and Li WP: Protective effect and mechanism of astragaloside IV on oxidative stress injury of mesangial cells. Zhongguo Zhong Yao Za Zhi 38: 725-730, 2013 (In Chinese)

43. Ha H, Hwang IA, Park JH and Lee HB: Role of reactive oxygen species in the pathogenesis of diabetic nephropathy. Diabetes Res Clin Pract 82 (Suppl 1): S42-S45, 2008.

44. Hodgkinson AD, Bartlett T, Oates PJ, Millward BA and Demaine AG: The response of antioxidant genes to hyperglycemia is abnormal in patients with type 1 diabetes and diabetic nephropathy. Diabetes 52: 846-851, 2003.

45. Babior BM: NADPH oxidase. Curr Opin Immunol 16: 42-47, 2004.

46. Gorin Y, Ricono JM, Kim NH, Bhandari B, Choudhury GG and Abboud HE: Nox4 mediates angiotensin II-induced activation of Akt/protein kinase B in mesangial cells. Am J Physiol Renal Physiol 285: F219-F229, 2003.

47. Devaraj S, Cheung AT, Jialal I, et al: Evidence of increased inflammation and microcirculatory abnormalities in patients with type 1 diabetes and their role in microvascular complications. Diabetes 56: 2790-2796, 2007.

48. Gruden G, Setti G, Hayward A, et al: Mechanical stretch induces monocyte chemoattractant activity via an NF-kappaB-dependent monocyte chemoattractant protein-1-mediated pathway in human mesangial cells: inhibition by rosiglitazone. J Am Soc Nephrol 16: 688-696, 2005.

49. Iwamoto M, Mizuiri S, Arita $M$ and Hemmi H: Nuclear factor-kappaB activation in diabetic rat kidney: evidence for involvement of P-selectin in diabetic nephropathy. Tohoku J Exp Med 206: 163-171, 2005.

50. Bäumer AT, Ten Freyhaus H, Sauer H, et al: Phosphatidylinositol 3-kinase-dependent membrane recruitment of Rac-1 and $\mathrm{p} 47^{\text {phox }}$ is critical for alpha-platelet-derived growth factor receptor-induced production of reactive oxygen species. J Biol Chem 283: 7864-7876, 2008

51. Graham S, Gorin Y, Abboud HE, et al: Abundance of TRPC6 protein in glomerular mesangial cells is decreased by ROS and PKC in diabetes. Am J Physiol Cell Physiol 301: C304-C315, 2011. 